\title{
NONLINEAR STABILITY OF SHOCK WAVES FOR VISCOUS CONSERVATION LAWS
}

\author{
BY TAI-PING LIU ${ }^{1}$
}

Consider the viscous conservation laws

$$
\frac{\partial u}{\partial t}+\frac{\partial f(u)}{\partial x}=\frac{\partial}{\partial x}\left(B(u) \frac{\partial u}{\partial x}\right), \quad t \geq 0,-\infty<x<\infty,
$$

where $u=u(x, t) \in \mathbf{R}^{n}$, the flux $f(u)$ is a smooth $n$-vector-valued function, and the viscosity $B(u)$ is a smooth $n \times n$ matrix. We are interested in the stability of traveling waves, the "viscous shock waves", for (1). It is shown that when the initial data are a perturbation of viscous shock waves, then the solution converges to these viscous shock waves, properly translated in space, in the uniform sup norm as time $t$ tends to infinity. Our analysis is based on the observation that a general perturbation also gives rise to diffusion waves in addition to translating viscous shock waves. A new technique combining the characteristic method and the energy method is introduced for the stability analysis. The energy method is a standard technique for parabolic systems. We use the method of characteristics, usually associated with hyperbolic systems, because, physically, the viscious shock waves and nonlinear diffusion waves are nonlinear hyperbolic waves in some general sense. This characteristic-energy method is based on a new understanding of nonlinear diffusion waves and, in particular, on their characterization as compression waves and weak expansion waves.

We assume that the associated hyperbolic conservation laws

$$
\frac{\partial u}{\partial t}+\frac{\partial f(u)}{\partial x}=0, \quad u \in \mathbf{R}^{n}
$$

are strictly hyperbolic; that is, $\partial f(u) / \partial u$ has real and distinct eigenvalues $\lambda_{1}(u)<\lambda_{2}(u)<\cdots<\lambda_{n}(u)$ :

$$
\begin{gathered}
\frac{\partial f(u)}{\partial u} r_{\imath}(u)=\lambda_{\imath}(u) r_{\imath}(u), \\
l_{\imath}(u) \frac{\partial f(n)}{\partial u}=\lambda_{i}(u) l_{\imath}(u), \quad i=1,2, \ldots, n .
\end{gathered}
$$

We assume that each characteristic field is either genuinely nonlinear or linearly degenerate $[\mathbf{5}]$. The behavior of shock waves, $N$-waves, and linear waves for (2) is well understood. It has been shown that a perturbation of shock waves gives rise to $N$-waves and linear waves. $N$-waves are described qualitatively by $N$-waves for the scalar equation

Received by the editors November 5, 1984 and, in revised form, December 21, 1984. 1980 Mathematics Subject Classification. Primary 35K55, 76N10; Secondary 35B40, 35L65. ${ }^{1}$ Partially supported by NSF Grant No. MCS 84-01355. 


$$
\begin{gathered}
\frac{\partial u}{\partial t}+u \frac{\partial u}{\partial x}=0, \quad u \in \mathbf{R}^{1}, \\
u(x, t)= \begin{cases}x / t & \text { for }-\sqrt{-2 p t} \leq x / t \leq \sqrt{2 q t} \\
0 & \text { otherwise }\end{cases}
\end{gathered}
$$

for time invariants $p$ and $q, p \leq 0 \leq q$. The linear waves are described by

$$
\frac{\partial u}{\partial t}+c \frac{\partial u}{\partial x}=0, \quad u \in \mathbf{R}^{1}
$$

where $c$ is a constant $[6]$.

For the system (1) a perturbation of viscous shock waves gives rise to nonlinear diffusion waves and linear diffusion waves. Our construction of nonlinear diffusion waves is based on the self-similar solutions for Burger's equation

$$
\begin{gathered}
\frac{\partial u}{\partial t}+u \frac{\partial u}{\partial x}=\alpha \frac{\partial^{2} u}{\partial x^{2}}, \quad u \in \mathbf{R}^{1} \\
u(x, t)=\left(\exp \left(\frac{\delta}{2 \sqrt{\alpha}}\right)-1\right) t^{-1 / 2} \exp \left(\frac{-x^{2}}{4 \alpha t}\right) \\
\cdot\left[\frac{2 \sqrt{\pi}}{\sqrt{\alpha}}+\left(\exp \left(\frac{\delta}{2 \sqrt{\alpha}}\right)-1\right) \int_{x / 2 \sqrt{\alpha t}}^{\infty} \frac{1}{\sqrt{\alpha}} \exp \left(-\xi^{2}\right) d \xi\right]^{-1} .
\end{gathered}
$$

The linear diffusion waves are constructed using the kernel for the heat equation

$$
\begin{gathered}
\frac{\partial u}{\partial t}+c \frac{\partial u}{\partial x}=\alpha \frac{\partial^{2} u}{\partial x^{2}}, \quad u \in \mathbf{R}^{1}, \\
u(x, t)=\delta(2 \pi \alpha t)^{-1 / 2} \exp \left(-(x-c t)^{2} / 4 \alpha t\right) .
\end{gathered}
$$

For the construction of the diffusion waves for (1) we require

$$
\alpha_{\imath}(u) \equiv l_{\imath}(u) B(u) r_{\imath}(u)>0, \quad i=1,2, \ldots, n .
$$

Condition (7) is satisfied for the compressible Navier-Stokes equations.

There is an essential difference between the elementary waves for (1) and (2) that is reflected in the difference between (3) and (5) and also between (4) and (6). For shock waves the difference vanishes as the vicosity tends to zero. On the other hand, the difference between the diffusion waves, (5) and (6), and $N$-waves and linear waves, (3) and (4), does not vanish as the viscosity tends to zero. In particular, the compressible Euler equations are qualitatively different from the compressible Navier-Stokes equations with regard to diffusion waves.

Our main result is

THEOREM. Suppose that the matrix

$$
\left(l_{1}(u), \ldots, l_{n}(u)\right)^{\mathrm{t}} B(u)\left(r_{1}(u), \ldots, r_{n}(u)\right)
$$

is positive definite for all $u$ under consideration. Then weak viscous shock waves for (1) are nonlinearly stable. More precisely, given weak viscous $i$ shock waves $\phi_{\imath}\left(x-\sigma_{\imath} t\right), i \in P \equiv\left\{P_{1}, P_{2}, \ldots, P_{l}\right\} \leq\{1,2, \ldots, n\}, 1 \leq P_{1}<$ $P_{2}<\cdots<P_{l} \leq n, \phi_{\imath}(-\infty) \equiv \bar{u}_{\imath-1}, \phi_{\imath}(+\infty) \equiv \bar{u}_{\imath}, i \in P$, and given $a$ 
weak perturbation $\bar{u}(x, 0)$, which tends to zero sufficiently fast as $t \rightarrow \pm \infty$, the solution $u(x, t)$ of $(1)$ and

$$
u(x, 0)=\sum_{\imath \in P} \phi_{\imath}(x)+\bar{u}(x, 0)
$$

exist globally in time and tend to the set of translated viscous shock waves $\phi_{\imath}\left(x+x_{\imath}-\sigma_{\imath} t\right):$ i.e.,

$$
\lim _{t \rightarrow \infty} \sup _{-\infty<x<\infty}\left|u(x, t)-\sum_{\imath \in P} \phi_{\imath}\left(x+x_{\imath}-\sigma_{\imath} t\right)\right|=0 .
$$

Moreover, the translations $x_{\imath}, i \in P$, are uniquely determined by

$$
\begin{aligned}
\int_{-\infty}^{\infty} \bar{u}(x, 0) d x \equiv \sum_{\imath \in P} x_{\imath}\left(\bar{u}_{\imath}-\bar{u}_{\imath-1}\right)+\sum_{\imath \notin P} \delta_{\imath} r_{\imath}\left(u_{0}\right) \\
r_{\imath}\left(u_{0}\right)=r_{\imath}\left(\bar{u}_{P_{\jmath}}\right) \text { for } P_{\jmath}<i<P_{\jmath+1} .
\end{aligned}
$$

For Burger's equation (5) the stability of viscous shock waves was proved by Hopf [2], using an explicit formula, and by Il'in-Oleinik [3], using the maximum principle, for the general scalar viscous conservation law (1), $u \in \mathbf{R}^{1}$. There had been no progress on the stability of viscous shock waves for general systems (1) until recently. Independently, Goodman [1] and MatzumuraNishihara [7] used the energy method to show that a single viscous shock wave is nonlinearly stable. Both papers made a stringent assumption that the perturbation has zero integral

$$
u(x, 0)=\phi(x)+\bar{u}(x, 0), \quad \int_{-\infty}^{\infty} \bar{u}(x, 0) d x=0 .
$$

It follows from the present study that the above assumption does not allow the presence of diffusion waves in the asymptotic state. The result in [7] for the equations of isentropic gas dynamics has been generalized to other systems by Kawashima-Matzumura [4], again under hypothesis (10).

Assumption (8) is not satisfied in the important case of the compressible Navier-Stokes equations. However, we expect that our technique can be refined to yield the nonlinear stability of viscous shock waves for the compressible Navier-Stokes equations.

\section{REFERENCES}

1. J. Goodman, Nonlinear asymptotic stability of vnscous shock profiles for conservation laws (preprint).

2. E. Hopf, The partial differential equation $u_{t}+u u_{x}=\mu u_{x x}$, Comm. Pure Appl. Math. 3 (1950), 201-230.

3. A. M. Il'in and O. A. Oleinik, Asymptotic behavor of the solutions of the Cauchy problem for certain quasilinear equations for large time, Mat. Sb. 51 (1960), 191-216. (Russian)

4. S. Kawashima and A. Matzumura, Asymptotic stabrlity of traveling wave solutions of system for one-dimensional gas motion. 
5. P. D. Lax, Hyperbolic systems of conservation laws. II, Comm. Pure Appl. Math. 10 (1957), 537-566.

6. T.-P. Liu, Linear and nonlinear large-time behavior of solutions of general systems of hyperbolic conservation laws, Comm. Pure Appl. Math. 30 (1977), 767-796.

7. A. Matzumura and K. Nishihara, On a stability of traveling wave solutions of a onedimensional model system of compressible viscous gas (preprint).

Department of Mathematics, University of MARYland, College Park, MARYLAND 20742 